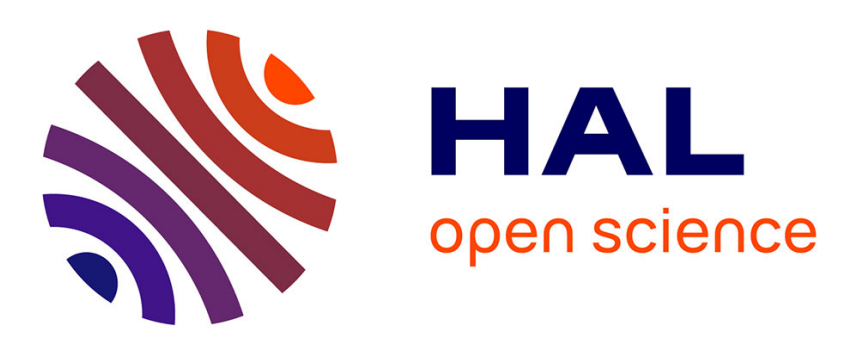

\title{
Vehicle to Pedestrian Communications for Protection of Vulnerable road Users
}

\author{
José Javier Anaya, Pierre Merdrignac, Oyunchimeg Shagdar, Fawzi \\ Nashashibi, José E. Naranjo
}

\section{- To cite this version:}

José Javier Anaya, Pierre Merdrignac, Oyunchimeg Shagdar, Fawzi Nashashibi, José E. Naranjo. Vehicle to Pedestrian Communications for Protection of Vulnerable road Users. 2014 IEEE Intelligent Vehicles Symposium, Jun 2014, Dearborn, Michigan, United States. pp.1-6. hal-00992759

\section{HAL Id: hal-00992759 https://hal.science/hal-00992759}

Submitted on 19 May 2014

HAL is a multi-disciplinary open access archive for the deposit and dissemination of scientific research documents, whether they are published or not. The documents may come from teaching and research institutions in France or abroad, or from public or private research centers.
L'archive ouverte pluridisciplinaire HAL, est destinée au dépôt et à la diffusion de documents scientifiques de niveau recherche, publiés ou non, émanant des établissements d'enseignement et de recherche français ou étrangers, des laboratoires publics ou privés. 


\title{
Vehicle to Pedestrian Communications for Protection of Vulnerable Road Users
}

\author{
José Javier Anaya ${ }^{1}$, Pierre Merdrignac ${ }^{2,3}$, Oyunchimeg Shagdar ${ }^{2}$, Fawzi Nashashibi ${ }^{2}$ and José E. Naranjo ${ }^{1}$
}

\begin{abstract}
Vehicle and pedestrian collisions often result in fatality to the vulnerable road users, indicating a strong need of technologies to protect such vulnerable road users. Wireless communications have potential to support road safety by enabling road users to exchange information. In contrast to vehicle to vehicle (V2V) and vehicle to infrastructure (V2I) communications for avoidance of inter-vehicle collisions, very limited efforts are made on communication mechanisms for pedestrian safety. This paper addresses the issue in a concrete way. We first formulate the requirement of the minimum information exchange distance for providing road users to have the necessary amount of time to perceive the situation and react. We then report our field tests and measurement based analysis to investigate if a Wi-Fi system can satisfy the application requirement. We also introduce a pedestrian protection application, V2ProVu, which provides the functionalities of the $\mathrm{Wi}-\mathrm{Fi}$ communications, risk calculation, and hazard alarming. Our study discloses several useful insights including 1) information exchange for a velocity of $80 \mathrm{~km} / \mathrm{h}$ has to be made before vehicle to pedestrian (V2P) distance is below 72 meters and 2) while this requirement is not too hard for radio communications technologies, the V2P communication range is greatly reduced if the signal is blocked by a human body.
\end{abstract}

\section{INTRODUCTION}

Vulnerable Road Users are defined as "non-motorized road users, such as pedestrians and cyclists as well as motorcyclists and persons with disabilities or reduced mobility and orientation" [1]. According to the statistics [2], more than 3000 people die daily due to dangerous driving, and half of them are vulnerable road users. The main reason of accidents is the inability of road users to detect and perceive oncoming dangers before a sufficient amount of time so that reactions for accident avoidance can be taken. Much attention has been put in detecting pedestrians and predicting the possibility of collisions using sensors and computer vision techniques [3]. Such sensor based approaches however do not perform well in poor visibility conditions e.g., at night time, in bad weather conditions, or the pedestrian is not close enough (e.g. within few tens meters) or in non-line-of-sight (NLOS) position w.r.t the sensor [3]. Therefore it is necessary to study and develop different technologies to fill the missing gap towards zero fatalities. One of such technologies is the radio

\footnotetext{
${ }^{1}$ José Javier Anaya, José E. Naranjo are with Escuela Universitaria de Informática, Universidad Politécnica de Madrid, Campus Sur Ctra. Valencia km. 728031 Madrid, SPAIN. $\{j j$.anaya, joseeugenio.naranjo\}@upm.es

2 Pierre Merdrignac, Oyunchimeg Shagdar, Fawzi Nashashibi are with IMARA Project-Team, INRIA Rocquencourt, Domaine de Voluceau, B.P. 105, 78153, Le Chesnay, FRANCE. name. surnamedinria.fr

3 Pierre Merdrignac is with Institut VeDeCoM, 77, rue des Chantiers, 78000, Versailles, FRANCE.
}

communications technology that has a potential to provide an extended "view" to the road users even in NLOS conditions.

Although safe driving support is one of the hot $R \& D$ subjects in the telecommunications field, the majority of the work has a focus on the V2V and V2I communications for avoidance of inter-vehicle collisions. An important achievement of these activities is the specification of the IEEE 802.11p amendment, Wi-Fi dedicated to ITS applications [4]. However, we believe that it is realistic to apply a technology, which is available in handheld devices, for V2P communications. Indeed, studies on the applicability of such technologies to the V2P communications have been carried out [5], [6]. The authors of [5] and [6] independently investigated the delay performances of $\mathrm{Wi}-\mathrm{Fi}$ and cellular systems and showed that $\mathrm{Wi}-\mathrm{Fi}$ can provide at least one order of magnitude shorter delay than that of the cellular systems (10 and $400 \mathrm{~ms}$ of delay in Wi-Fi and 3G systems, respectively [6]). An important lesson provided by [5] and [6] is that without denying the possibilities of using both the systems in a combined way, Wi-Fi seems to be the primary choice especially for low-latency direct V2P communications.

To this end, we study Wi-Fi based V2P communications for pedestrian safety support. Vehicle and pedestrian collision can be avoided by informing the driver of the existence of the pedestrians and/or by informing the pedestrians of the existence of the oncoming vehicles. The majority of the existing work on computer vision and telecommunications fields [3], [5], [7] targets the former approach. We believe that for zero fatalities, it is also necessary that pedestrians have information and react for their own safety. Therefore, leaving the former approach to our future work, this paper focuses on the latter approach i.e., vehicles inform pedestrians of their existence. Since a very limited effort is made on V2P communications for pedestrian safety, we address the issue in a concrete way. Specifically, we first formulate the requirement on the minimum information exchange distance for allowing pedestrians to have necessary time to perceive and react. Because the minimum distance is a function of the positioning accuracy and the communication range, we carry out real-world experiments and investigate the performances. The GPS measurements show that $10 \mathrm{~m}$ of positioning error should be expected even in a good weather condition. Although, connection time for V2P communications might be long, we assume vehicles and pedestrians have initially been connected to the same network and are exchanging information within this network. Hence, the Wi-Fi performances are measured in terms of packet delivery ratio (PDR) 
and packet inter-reception time (PIR) for different scenarios, where the pedestrian carries the handheld device in different manners. The results imply that communication coverage can be reduced by a hundred meter if the radio signal is blocked by the human body. Nevertheless, we show that Wi-Fi can satisfy the application requirement with a condition that the transmission frequency is higher than $1 \mathrm{~Hz}$. Finally, we developed an application, V2ProVu, for vehicle on-board and pedestrian handheld devices providing functionalities of the Wi-Fi communication, risk calculation, and hazard alarming. For the risk calculation and hazard alarming, we define a geographical area (GDA: geographical destination area) w.r.t the vehicle on which pedestrians have to be informed of the oncoming vehicle.

The paper is organized as follows. Section II highlights the related work. Section III defines the application requirement followed by performance investigation of V2P communication system in Section IV. Section V introduces the calculation of GDA and our application, V2ProVu, which is developed to investigate the feasibility of pedestrian safety supported by V2P communications. Finally, Section VI concludes our paper.

\section{RELATED WORK}

Pedestrian safety has been an issue for many years in the automotive field. Solutions based on vehicle embedded sensors (e.g. camera, laser, and radar) and computer vision technologies have been proposed [3], [8]. Reference [9] introduces Walk-Safe application, which detects oncoming vehicles using the camera embedded in smartphones. As mentioned earlier, sensor and computer vision techniques have strong constraints regarding sensor locations (for example Walk-Safe cannot be used if the smartphone is e.g., in a pocket or if the camera is facing to the ground or the sky), coverage and field of view.

Projects such as WATCH-OVER [7] and Ko-TAG [10] rely on pedestrian to vehicle communications to enhance safety. Within this projects, methods based on radio signal propagation have been applied to estimate precisely V2P distance. However, such methods require the development of specific electronic devices for pedestrians who are already carrying handheld devices with many capabilities. The authors of [11] carried out a detailed study targeting the GPS receivers embedded in handheld devices and concluded that assistance can only be given in scenarios with low requirements on the GPS accuracy. Therefore, we considered performances of such GPS systems in our work.

In August 2013, Honda announced its development of a V2P communications technology based on DSRC (Dedicated Short Range Communication) on $5.9 \mathrm{GHz}$ for pedestrian safety [12]. Assuming that the mentioned DSRC is the IEEE 802.11p, a use of the IEEE 802.11p for V2P is somehow questionable because 1) users already carry handheld devices which have the capabilities of short, medium, and long range communications (Bluetooth, Wi-Fi, and 3/4G) and 2) the frequency channel of IEEE 802.11p (especially the control channel, which is for safety applications) is expected to have a serious channel congestion due to $\mathrm{V} 2 \mathrm{~V}$ communications in a dense road environment. Nevertheless, the IEEE 802.11p, is a Wi-Fi technology, and hence we believe that our study, which is carried out using the IEEE $802.11 \mathrm{~g}$, is generally valid for the IEEE 802.11p too. General Motors is developing a V2P communications system based on WiFi-Direct which is already available in smartphones [13]. Unfortunately none of [12] and [13] provided information about their mechanisms to protect vulnerable road users.

The authors of [5] and [6] investigated the performances of Wi-Fi and cellular systems, and show that Wi-Fi can show significantly shorter communications delay. Furthermore, [5] defined a requirement to $\mathrm{V} 2 \mathrm{X}$ communications for road safety applications, assuming that positioning is perfectly accurate. We extend the requirement for V2P communications with no assumption on positioning accuracy. The authors of [6] proposed a mechanism to use both of the cellular and Wi-Fi systems for pedestrian safety. The key player of the mechanism is a control centre that communicates with vehicles and pedestrians using the cellular network. If the control centre detects a risk of collision between a pedestrian and a vehicle, it asks the pair of vehicle and pedestrian to communicate directly using Wi-Fi. We also believe the number of traffic accidents can be greatly reduced by using different communication systems. However the proposed approach in [6] is too much "centralized" so that processing and communications at the control centre could be the bottleneck of the overall system, resulting in a scalability problem. Most importantly, because a very little is known about the applicability of Wi-Fi for pedestrian safety, we decided to first focus on Wi-Fi systems. The key contributions of our work in contrast to the existing work are as follows: 1) we formulated the application requirement for the pedestrian safety 2) we investigated the GPS and Wi-Fi performances and achieved useful insights especially regarding the impact of the blockage by a human body 3) we carried out measurement-based analysis to investigate if the Wi-Fi system can satisfy the application requirement, and finally 5) we developed an application with the functionalities of Wi-Fi communications, risk calculation, and hazard alarming.

\section{APPlication ReQuirement}

Similar to inter-vehicle communications, collisions between vehicles and pedestrians can be largely avoided by enabling vehicles to periodically broadcast information on their position, velocity, moving direction, and etc. ETSI defined such a type of messages as Cooperative Awareness Message (CAM). In this section, we define the application requirement to CAM delivery in $\mathrm{V} 2 \mathrm{P}$ scenarios.

The authors of [5] defined system available time $t_{\text {sta }}$ which refers to the remaining time excluding that for communications, driver's perception and reaction. Since we are interested in the case, where pedestrians receive CAMs and react accordingly, we re-define $t_{\text {sta }}$ :

$$
t_{s t a}=\frac{d}{v}-t_{p}-t_{r},
$$


where $v$ is the velocity of the vehicle and $d$ is the distance between the vehicle and the pedestrian at the time, when the pedestrian receives a CAM from the vehicle. $t_{p}$ and $t_{r}$ are the time required for perception (i.e., the pedestrian recognizes the existence of the oncoming vehicle) and reaction. Obviously, for collision avoidance, it is required that $t_{s t a}>0$.

It is realistic to expect that in order to estimate collision risk, upon reception of a CAM, the pedestrian (the device) calculates the distance $(d)$ based on the position information included in the CAM and its own position. Therefore the application is largely dependent on the accuracy of the position information and the transmission delay of the V2P communi-

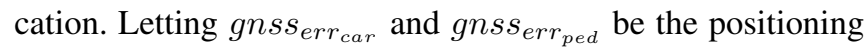
error at the vehicle and the pedestrian, respectively, and $t_{t x}$ be the transmission delay, the pedestrian may over-estimate the distance: $d=d_{c a l}-g n s s_{e r r_{c a r}}-g n s s_{e r r_{p e d}}-v \times t_{t x}$, where $d_{c a l}$ is the calculated distance based on the GPS information. Finally, following $t_{s t a}>0$, we get $d_{c a l}>d_{\text {min }}$, where

$$
\begin{gathered}
d_{\text {min }}=v \times\left(t_{p}+t_{r}+t_{t x}\right)+g n s s_{\text {err }_{\text {car }}}+g n s s_{\text {err }} \text { ped } \\
\text { IV. PERFORMANCE INVESTIGATION OF V2P } \\
\text { COMMUNICATIONS SYSTEM }
\end{gathered}
$$

Motivated by the application requirement, we investigate the GPS accuracy and the PDR and PIR of performances of the IEEE 802.11g.

\section{A. System description}

Vehicle to pedestrian communications are evaluated using a Citroen $\mathrm{C} 1$, equipped with a communication device, NexCom, which is installed with the IEEE 802.11g and a conventional GPS chip. The vehicle also has a separate Real Time Kinematic (RTK) GPS receiver, which is used as a ground-truth for the vehicle's positioning (see Fig. 1). The pedestrian carries Samsung Galaxy Tab2 10.1 with version 4.0.4 of Android OS.

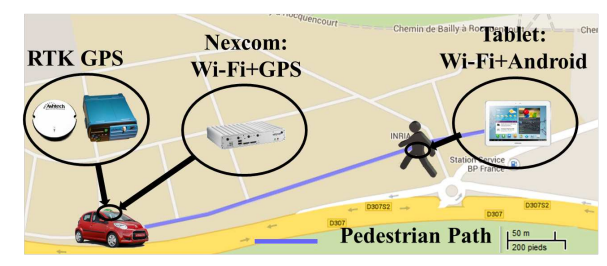

Fig. 1: Experimental setup.

\section{B. GPS Accuracy}

The worst scenario regarding position information is probably the case, where no GPS information is available due to e.g., a bad weather condition. We investigate rather a "good-case" positioning performance, i.e., the accuracy of a "conventional" GPS on a sunny day. Specifically, we run Citroen $\mathrm{C} 1$ at Inria site, collected the position information provided by the GPS receiver of NexCom, and compared it with that of RTK GPS, whose positioning information is corrected by a reference station, providing centimeter-level accuracy.

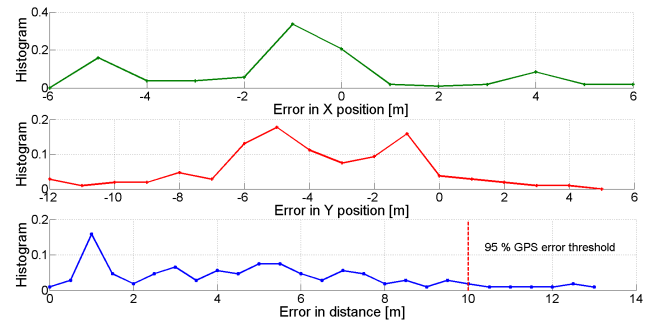

Fig. 2: Histogram of GPS error in longitudinal and lateral position, and in distance

Figure 2 shows the histogram of the positioning error in terms of longitudinal and lateral positions, and distance. Similarly to the authors of [11], we notice that GPS error on lateral direction is larger than that of longitudinal. Besides, if we consider $95 \%$ of the GPS error in distance, then we determine GPS error is approximately $10 \mathrm{~m}$. To this end, our experiments confirmed that the GPS devices of vehicles' onboard systems and pedestrians' hand-held show GPS error that is not generally satisfactory for safety applications, even in "good case" conditions.

\section{Wi-Fi Performance}

In order to collect a sufficient amount of data for varying distances, the vehicle is parked on a road and the pedestrian walks away from the vehicle (at around $1 \mathrm{~m} / \mathrm{s}$ speed) following the 500 meters road as illustrated in Fig. 1. An initital connection is established between the vehicle and the pedestrian. Because a conventional GPS provides position update every $1 \mathrm{~s}$, the vehicle broadcasts 110 Bytes of packets every 1 second with $12.0 \mathrm{Mbps}$ coding rate at $27 \mathrm{dBm}$ transmission power. The tablet of the pedestrian collects the received packets for investigation of PDR and PIR performances. The performance measurements are made for the following two scenarios.

- Scenario 1: Pedestrian holds the tablet in his front close to his body. In this scenario, the human body blocks the signal emitted from the vehicle.

- Scenario 2: Pedestrian carries the tablet in his backpack, resulting in the signal is blocked only by the cotton tissue of the backpack.

1) Packet Delivery Ratio (PDR): Figure 3 shows the packet delivery performance for the two scenarios. As can be seen in the figure, the V2P communication is achievable over 400 meters of distance. PDR at $400 \mathrm{~m}$ is only $30 \%$ for scenario 1 , whereas it is $60 \%$ for scenario 2 , clearly indicating the significant impact of the signal blockage by the human body. Finally, as we expect, PDR degrades with the increase of the distance for both the scenarios. The results show that in order to achieve e.g., $80 \%$ of PDR, the distance needs to be smaller than $130 \mathrm{~m}$ (resp. $305 \mathrm{~m}$ ) for scenario 1 (resp. scenario 2).

2) Packet Inter Reception (PIR) Time: In our experiments, we observed very short transmission delay (appx. $10 \mathrm{~ms}$ ) without depending on the V2P distance. An important performance metric for safety applications is packet inter-reception 


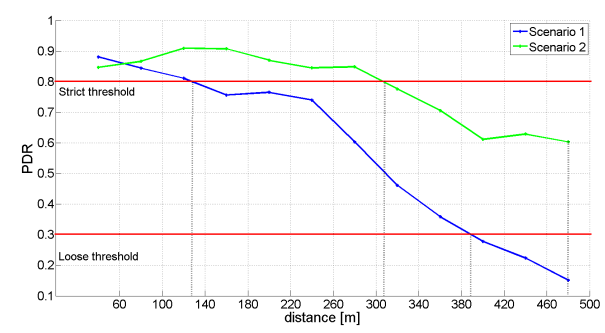

Fig. 3: Packet Delivery Ratio (PDR)

time (PIR), indicating the time interval during which receiver does not have an information update [14]. Because the transmission delay is significantly smaller than the packet transmission interval ( $1 \mathrm{~s}$ ), PIR is approximately $k \mathrm{~s}$, where $k$ is an integer. As shown in Figures 4(a) and 4(b), based on our experimental results, we calculate the cumulative distribution function $p\left(P I R>k \mid d>d_{t h}\right)$ for the two scenarios. The function can be interpreted as the probability of not receiving more than $k$ consecutive CAMs, when the $\mathrm{V} 2 \mathrm{P}$ distance is larger than $d_{t h}$ (note that the horizontal axis of the figures are $\left.d_{t h}\right)$. As the results show that $p\left(P I R>k \mid d>d_{t h}\right)$ increases with the increase of $d_{t h}$ and it is larger for smaller $k$. Finally, we observe that the probability of not receiving more than 6 consecutive CAMs is very small, and hence it can be neglected.

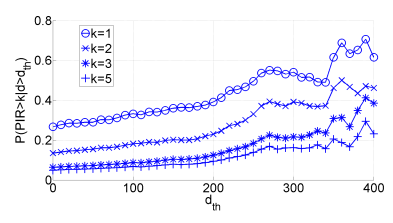

(a) Scenario 1 .

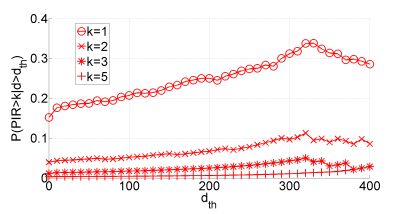

(b) Scenario 2 .
Fig. 4: Probability of not receiving more than k consecutive packets.

\section{Measurement based study on the applicability of Wi-Fi for pedestrian protection}

Based on our experimental results, we first calculate the minimum required information exchange distance, $d_{\min }$ (see Eq. (2)). According to our results and that presented in [11],

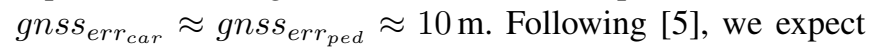
human needs appx. $0.83 \mathrm{~s}$ to perceive the "meaning" of the information provided by the V2P communications system $\left(t_{p}=0.83 \mathrm{~s}\right)$. The reaction time, i.e., the time required to react (e.g., avoid) depends on the situation. However, for the worst case, we can imagine that the pedestrian was in the middle of the road and $t_{r}$ is the time required to cross the half of the road. Therefore, setting the road width to e.g., $3 \mathrm{~m}$ and the velocity of the pedestrian to $1 \mathrm{~m} / \mathrm{s}$, the reaction time, $t_{r}=1.5 \mathrm{~s}$. Moreover, according to our experiments and the results provided in [6], the transmission delay is approximately $10 \mathrm{~ms}$. Wrapping everything up, we get $d_{\text {min }}$ is equal to $39.5,52.3$, and $72.0 \mathrm{~m}$, when the velocity of the vehicle is $30,50,80 \mathrm{~km} / \mathrm{h}$, respectively.
Inspired by [14], we take a measurement-based analysis to investigate whether the Wi-Fi system can satisfy the application requirement. Since vehicles periodically broadcast CAMs, we can say that the transmission of message $i$ is occurred at the V2P distance, $d_{i}$. Therefore the message reception status at a given $d$, where $d_{i+1}>d>d_{i}$ (note that if $i>j$, then $d_{i}>d_{j}$ ), is same as that at $d_{i+1}$. Now it is realistic to define that at a given V2P distance $d$ $\left(d_{i+1}>d>d_{i}\right)$, if none of the $k$ consecutive messages, $i+k, i+k-1, \ldots i+2, i+1$, is received, then the pedestrian is not "informed" of the existence of the vehicle. Since the probability of message reception at $d_{i}$ is $\operatorname{PDR}\left(d_{i}\right)$, the probability of being informed at $d_{\min }$ is

$$
p\left(d_{\text {min }}\right)=1-\bar{p}\left(d_{\text {min }}\right)=1-\prod_{i=1}^{k}\left(1-\operatorname{PDR}\left(d_{i}\right)\right),
$$

where

$$
d_{i}=d_{\min }+\frac{v \times i}{f_{t x}} .
$$

Here $f_{t x}$ is the CAM generation frequency. It should be noted that since we cannot expect that a transmission occurs at exactly $d_{i}$ (in (4)), the $P D R\left(d_{i}\right)$ in (3) is the average PDR corresponding to the range $\left.] d_{i-1}, d_{i}\right]$.

Figure 5 draws the probability of being informed at $d_{\text {min }}$ for different values of $k$. Here, $f_{t x}$ is $1 \mathrm{~Hz}$. As can be seen in the figure, the probability of being informed is very high (larger than 0.8 ) for any speed and scenario. In order to have 1 , however, $k$ needs to be larger than 3 , implying a need for increased transmission frequency. We end this section by concluding that 1) Wi-Fi can be used for V2P communications for pedestrian safety; 2) for further performance improvement, the message generation frequency has to be larger than $1 \mathrm{~Hz}$.

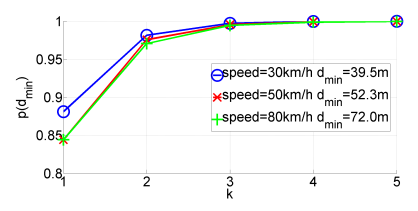

(a) Scenario 1 .

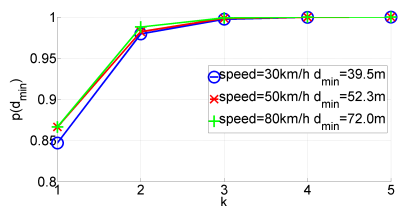

(b) Scenario 2 .
Fig. 5: Probability of being informed at $d_{\min }$.

\section{V2ProVu: Developing of Wi-Fi based PEDESTRIAN PROTECTION APPLICATION}

While it is important to alert pedestrians of oncoming dangers, an attention has to be paid to over-alarming. Specifically, pedestrians can receive messages from many vehicles around them. However, not all of the vehicles influence pedestrians' safety. Thus, messages sent by vehicles should be "filtered" such that a focus is given to only the vehicles, which have risks of collisions. For such message filtering, we define GDA, which is calculated based on the kinematics of the vehicle and indicates that the pedestrians in the GDA has a collision risk with the vehicle. This section defines GDA 
and introduces our application, V2ProVu, which uses GDA for risk calculation.

\section{A. Geographical Destination Area calculation}

GDA can be determined based on the vehicle's kinematics, especially, the vehicle's position, speed, and yaw rate, length, and width. It should be noted that speed and yaw rate can normally be obtained from the CAN bus of the vehicle, but they can be measured by external sensors or simply be calculated from the GPS information. The shape of GDA is different if the near-future moving direction of the vehicle is straight or turning as illustrated in Figure 6.

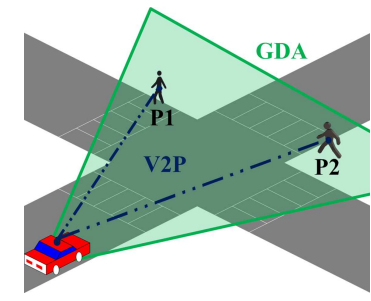

(a) Vehicle is moving straight.

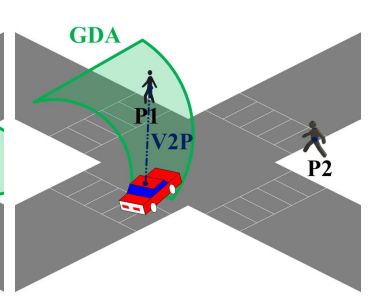

(b) Vehicle is turning.
Fig. 6: Geographical destination area (GDA) for straight and left-turning vehicles.

In order to determining GDA, first, the latitude and longitude positions of the vehicle and the pedestrian are transformed into in a cartesian coordinate system using usual world geodetic system (wgs84). Then, the vehicle's trajectory

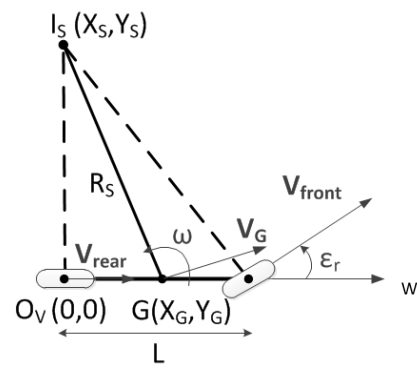

(a) Bicycle model

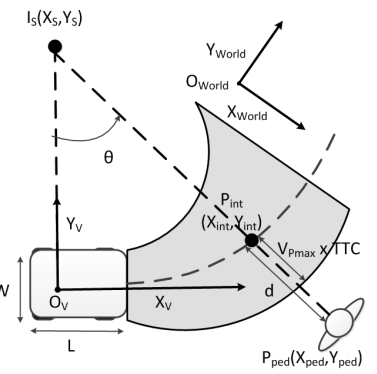

(b) Pedestrian alert system
Fig. 7: Model to calculate GDA.

is represented using the bicycle model (with 2 tires). A local coordinate system $\left(O_{V}, X_{V}, Y_{V}\right)$, which is centered at the rear tire is built as shown in figure 7(a). Based on the coordinate and the available information (specifically, velocity $\left(V=\left\|V_{G}\right\|\right)$ and yaw rate $(\omega)$ ), the radius to the gravity center $\left(R_{S}\right)$, the Ackerman angle (the vehicle steering angle $\epsilon_{r}$ ), and the Instantaneous Center of Rotation (ICR) $I_{S}\left(X_{S}, Y_{S}\right)$ can be found:

$$
\begin{array}{cc}
R_{S}=\frac{V}{\omega}, & \epsilon_{r}=\frac{L}{R_{S}} \\
X_{S}=0, & Y_{S}=R s \times \cos \left(\frac{\epsilon_{r}}{2}\right) .
\end{array}
$$

Finally, using the above-calculated values, the right and left boundaries of GDA (green lines in Fig. 6(a) and 6(b)) can be calculated following the parametric equation (due to the lack of space, the equations are not presented). Once a vehicle calculates the GDA, it can include a representation of the GDA in its CAM so that pedestrians determine whether they are in the GDA. However, as can be seen in Fig. 6(b), GDA can have a complicated shape, thus a representation of the shape and including the information in CAMs would increase processing and transmission overhead. Therefore, in our application, we took an equivalent approach, where pedestrians calculate time-to-collision (TTC) that determines if the pedestrians are in the GDA. The intersection point between the pedestrian and vehicle's trajectories, $P_{\text {int }}$, is calculated based on the vehicle's trajectory and the line that connects $I_{S}$ and the pedestrian position, $P_{\text {ped }}$ (see Figure 7(b)). Then, TTC, i.e., the time required for the vehicle to reach $P_{\text {int }}$, is calculated by applying the bicycle model to estimate the near-future trajectory. Finally, we consider that there is a risk of collision, if the pedestrian can reach $P_{\text {int }}$ before the vehicle arrives. In other words, i.e, letting $d$ be the distance between $P_{\text {int }}$ and $P_{\text {ped }}$, there is a risk of collision if $d<V_{p_{\max }} \times T T C$, where $V_{p_{\max }}$ is the typical value of the maximum velocity of pedestrians. The detail of the algorithm is provided in Algorithm 1.

\section{Algorithm 1 Pedestrian Alert System}

Input: $\mathrm{V}, \omega$ : Vehicle speed and yaw rate

$X_{\text {ped }}, Y_{\text {ped }}$ : Pedestrian position in vehicle coordinate system

Parameters: L, W: Vehicle length and width

$V_{p_{\max }}$ : Maximum pedestrian speed

Output: Packet is accepted or not

$$
\begin{aligned}
& \text { Calculate } R_{S}, \epsilon_{r}, X_{S} \text { and } Y_{S} \text { using Eq. (5) } \\
& \theta \leftarrow \operatorname{atan} 2\left(\frac{Y_{p e d}-Y_{S}}{R_{S}}, \frac{X_{p e d}-X_{S}}{R_{S}}\right) \\
& T T C \leftarrow \frac{\theta-\frac{\epsilon_{r}}{2}+\frac{\pi}{2}}{\omega} \\
& X_{\text {int }} \leftarrow R_{S} \times \cos \left(\theta+\frac{\epsilon_{r}}{2}-\frac{\pi}{2}\right)+X_{S} \\
& Y_{\text {int }} \leftarrow R_{S} \times \sin \left(\theta+\frac{\epsilon_{r}}{2}-\frac{\pi}{2}\right)+Y_{S} \\
& d \leftarrow \sqrt{\left(X_{\text {ped }}-X_{\text {int }}\right)^{2}+\left(Y_{\text {ped }}-Y_{\text {int }}\right)^{2}} \\
& \text { Accept if } d<V_{p_{\text {max }}} \times T T C
\end{aligned}
$$

\section{B. Application Design and Operational Test}

As a proof of concept we developed a Java application, called V2ProVu, which consists of the transmitter and receiver sides. The transmitter V2ProVu permanently collects information on the position and the kinematics of the vehicle and periodically generates CAMs and transmits over Wi$\mathrm{Fi}$ interface using UDP. The receiver V2ProVu receives the CAMs and if itself is in the GDA, it provides an alarm to the pedestrian. The transmitter V2ProVu is implemented in a Linux system (that of NexCom) and the receiver V2ProVu is implemented in the Android system of the Samsung Galaxy.

Furthermore, we also considered the timing of triggering alarms to the pedestrian. Let us imagine the scenario illustrated in Fig.6. In Fig.6(a), the vehicle's near-future trajectory is straight, therefore, the pedestrian, P2, was in the GDA. However, after some period of time (Fig. 6(b)), the vehicle changes its trajectory (turns left), resulting in P2 be out of 
GDA. Obviously, if the system triggers alarm as soon as the pedestrian is in the GDA, the system would cause many false alarms. Therefore, we divide a GDA to two sub-areas using TTC. If TTC is above a given threshold, $t_{t h}$, the pedestrian is only informed of the presence of the vehicle. However, if TTC is below $t_{t h}$, then the pedestrian receives an alert. We formulate $t_{t h}$ in Eq. (6) using the requirement defined in Section III, and $k_{t h}$, the number of consecutive packets that should be sent to ensure that the pedestrian is informed with a high probability (see Section IV-C).

$$
t_{t h}=t_{p}+t_{r}+t_{t x}+\frac{k_{t h}}{f_{t x}}
$$

Finally, we carried out a number of experiments to verify the functionalities implemented in V2ProVu. In the experiments, a vehicle, which is equipped with the V2ProVu transmitter, approaches a pedestrian who stands still on the side edge of the road holding the V2ProVu receiver (the tablet). Based on the results in Figure 5 we set $k_{t h}$ to 4 . With the values of $t_{p}, t_{r}, t_{t x}$ and $f_{t x}$ from Section IV-C, we find $t_{t h}=6.5 \mathrm{~s}$. Figure 8 illustrates one of our experiments. In the figure, the positions of the vehicle at the time, $t_{1}$, $t_{2}$, and $t_{3},\left(t_{1}<t_{2}<t_{3}\right)$ and the corresponding screen snapshots of the V2ProVu receiver are shown. At $t=t_{1}$, the distance is $65.31 \mathrm{~m}$ and the color of HMI is green, indicating the pedestrian is outside of the GDA. On the other hand, at $t=t_{2}$ (resp. $t=t_{3}$ ), the pedestrian is inside the GDA, the $\mathrm{V} 2 \mathrm{P}$ distance is $34.63 \mathrm{~m}$ (resp. $19.86 \mathrm{~m}$ ), TTC $=8.5 \mathrm{~s}$ (resp. TTC $=4 \mathrm{~s}$ ) which is above (resp. below) $t_{t h}$ and thus the HMI is yellow (resp. red), indicating a potential danger (resp. a high collision risk).

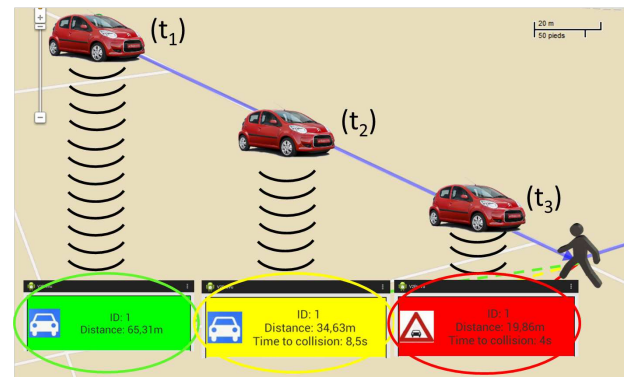

Fig. 8: Example of V2ProVu HMI for a vehicle approaching a pedestrian

\section{CONCLUSION}

Wireless communications technology has a potential to support safety of vulnerable road users by enabling information exchange between pedestrians and vehicles. This paper introduces our study on pedestrian safety support by Wi-Fi based V2P communications. We formulated the requirement of the minimum information exchange distance for V2P communications for pedestrian protection and conducted a study on the applicability of Wi-Fi based V2P communications system. The real-world experiments show that it is necessary to consider $10 \mathrm{~m}$ of GPS error even in a good weather condition. Moreover while $400 \mathrm{~m}$ of communication coverage is achievable, if the signal is blocked by the human body, the communications distance would be significantly shorter. Nevertheless, by measurement based analytical study, we showed that the Wi-Fi system can satisfy the application requirement, with the condition of increased transmission frequency (larger than $1 \mathrm{~Hz}$ ). Finally, V2ProVu, which is an application for pedestrian protection by providing the functionalities of $\mathrm{Wi}-\mathrm{Fi}$ communications, risk calculation, and hazard alarming, is introduced. Our future work includes an extended experimental study on pedestrian to vehicle communication mechanisms for safety of vulnerable road users. Moreover, we also plan to investigate the interference issue in the non-ISM band (e.g., 2.4 GHz) having in mind of the possibility of applying DSRC for V2P communications.

\section{ACKNOWLEDGMENT}

This work benefits from a financial support of French funding "financement par le plan d'Avenir" and Spanish national project TRA2010-15645.

\section{REFERENCES}

[1] Directive 2010/40/EU of the European Parliament and of the Council of 7 July 2010 on the framework for the deployment of Intelligent Transport Systems in the field of road transport and for interfaces with other modes of transport Text with EEA relevance, Std.

[2] WHO, "Global Status Report On Road Safety 2013: supporting a decade of action," World Health Organization, Tech. Rep., 2013.

[3] T. Gandhi and M. Trivedi, "Pedestrian Protection Systems: Issues, Survey, and Challenges," IEEE Transactions on Intelligent Transportation Systems, vol. 8, no. 3, pp. 413-430, Sept. 2007.

[4] IEEE Standard for Information technology - Telecommunications and information exchange between systems - Local and metropolitan area networks - Specific requirement, Part 11: Wireless LAN Medium Access Control (MAC) and Physical Layer (PHY) Specifications, IEEE Computer Society Std., July 2010, iEEE Std 802.11p-2010.

[5] K. David and A. Flach, "Car-2-x and pedestrian safety," Vehicular Technology Magazine, IEEE, vol. 5, no. 1, pp. 70-76, 2010.

[6] C. Sugimoto, Y. Nakamura, and T. Hashimoto, "Prototype of pedestrian-to-vehicle communication system for the prevention of pedestrian accidents using both $3 \mathrm{~g}$ wireless and wlan communication," in 3rd International Symposium on Wireless Pervasive Computing, 2008. IEEE, 2008, pp. 764-767.

[7] L. Andreone, A. Guarise, F. Lilli, D. M. Gavrila, and M. Pieve, "Cooperative Systems for vulnerable road users: The Concept Of The WATCH-OVER Project," in ITS World Congress, 2006.

[8] G. Gate, A. Breheret, and F. Nashashibi, "Centralized fusion for fast people detection in dense environment," in IEEE International Conference on Robotics and Automation, 2009., 2009, pp. 76-81.

[9] T. Wang, G. Cardone, A. Corradi, L. Torresani, and A. T. Campbell, "WalkSafe : A Pedestrian Safety App for Mobile Phone Users Who Walk and Talk While Crossing Roads Categories and Subject Descriptors," in HotMobile '12, San Diego, CA, USA, 2012.

[10] "Ko-tag - kooperative transponder." [Online]. Available: http: //ko-fas.de/deutsch/ko-tag---kooperative-sensorik.html

[11] M. Liebner, F. Klanner, and C. Stiller, "Active Safety for Vulnerable Road Users based on Smartphone Position Data," in 2013 IEEE Intelligent Vehicles Symposium, 2013.

[12] Honda demonstrates advanced vehicle-to-pedestrian and vehicle-to-motorcycle safety technologies. [Online]. Available: http://www.prnewswire.com/news-releases/honda-demonstratesadvanced-vehicle-to-pedestrian-and-vehicle-to-motorcycle-safetytechnologies-221495031.html

[13] $\mathrm{Gm}$ developing pedestrian safety technology that spots smartphones. [Online]. Available: http://www.caradvice.com.au/183936/ gm-developing-pedestrian-safety-technology-that-spots-smartphones/

[14] F. Martelli, M. E. Renda, G. Resta, and P. Santi, "A Measurementbased Study of Beaconing Performance in IEEE 802 . 11p Vehicular Networks," in 2012 Proceedings IEEE INFOCOM, 2012, pp. 15031511. 